\title{
Charge Transport in Organic Transistors Accounting for a Wide Distribution of Carrier Energies-Part II: TFT Modeling
}

\author{
Fabrizio Torricelli, Kevin O’Neill, Gerwin H. Gelinck, Kris Myny, Student Member, IEEE, \\ Jan Genoe, Member, IEEE, and Eugenio Cantatore, Member, IEEE
}

\begin{abstract}
A physically based analytical model of the drain current of an organic thin-film transistor is proposed. It is compared with the measurements collected from transistors made with different gate insulators, organic semiconductors, and fabrication processes. The extracted model parameters provide quantitative information on the charge transport in the active layer of the transistor. The analysis suggests that the tail states are an intrinsic property of the organic semiconductor, whereas the deep states arise from the interaction between the semiconductor and the gate insulator. The relative importance of tail and deep localized states is related to the operating regions of the transistor. The resulting mathematical expressions are simple and suitable for computer-aided design implementation.
\end{abstract}

Index Terms-Computer-aided design (CAD) applications, density of states (DOS), organic thin-film transistor (OTFT).

\section{INTRODUCTION}

$\mathbf{O}$ RGANIC electronics have been identified as a viable candidate for low-cost, disposable, large-area, and flexible electronics. The transistors are the main building blocks for any electronic circuit. Therefore, understanding and modeling the physical phenomena occurring in organic thin-film transistors (OTFTs) is essential to further improve the device performance and to rationally design organic circuits.

Over the years, many models to study and/or describe the charge transport in OTFTs have been proposed in the literature [1]-[7]. All of them take into account, directly or indirectly, the effect of the density of states (DOS) on the drain current. More in detail, in the pioneering work of Horowitz and Delannoy [1],

Manuscript received December 4, 2011; accepted January 12, 2012. This work was supported in part by the Dutch Technology Foundation STW, which is the applied science division of NWO, and in part by the Technology Programme of the Ministry of Economic Affairs. The review of this paper was arranged by Editor A. C. Arias.

F. Torricelli and E. Cantatore are with the Department of Electrical Engineering, Eindhoven University of Technology, 5600MB Eindhoven, The Netherlands (e-mail: F.Torricelli@tue.nl; fabrizio.torricelli@ing.unibs.it).

K. O'Neill is with Polymer Vision B. V., 5616LZ Eindhoven, The Netherlands.

G. H. Gelinck is with Holst Centre, 5656AE Eindhoven, The Netherlands.

K. Myny is with the Polymer and Molecular Electronics Group, IMEC, 3001 Leuven, Belgium, and also with the Katholieke Universiteit Leuven, 3001 Leuven, Belgium.

J. Genoe is with the Polymer and Molecular Electronics Group, IMEC, 3001 Leuven, Belgium, and also with the Katholieke Hogeschool Limburg, 3590 Diepenbeek, Belgium.

Color versions of one or more of the figures in this paper are available online at http://ieeexplore.ieee.org.

Digital Object Identifier 10.1109/TED.2012.2184764 the model was based on a single trap level located near the majority-carrier transport band. Subsequent works [2], [3] accounted for the effect of charge trapping by empirical gatevoltage-dependent mobility. Such dependence was physically explained by Vissenberg and Matters [8] assuming an exponential DOS and solving the variable-range hopping (VRH) transport by means of the percolation theory. The derived expressions of the conductivity and the mobility have been the starting point to develop several OTFT models recently proposed [4]-[7].

On the other hand, the assumption of an exponential DOS seems to be in contradiction with several specific studies [9][14], which extracted the DOS from thin-film transistors (TFTs) based on different organic semiconductors. These investigations demonstrate that the DOS of single-crystal, amorphous, doped, or undoped organic semiconductors can be well approximated by a Gaussian function, an exponential function, or by a combination of these functions. More specifically, the tail states can be approximated by a Gaussian or an exponential function, whereas the localized states at deep energies are well described by an exponential function.

In this paper, the theory proposed in Part I [15] is used to derive an analytical model for OTFTs with a double exponential DOS. The model is compared with a large number of experimental data collected from transistors made using different organic semiconductors, gate insulators, and fabrication processes. This analysis reveals the origin of the deep trap states and thus reconciles the different experimental results. Furthermore, the model enables a detailed discussion of the relative importance of deep and tail states on the measured characteristics, and the extracted parameters yield physical information about the charge carrier transport. A quantitative criterion is given in order to understand, directly from the experimental data, the usefulness of the proposed theory and model. The OTFT model is the natural extension of [4]-[7].

This paper is organized as follows: In Section II, transistor fabrication is described. In Section III, the analytical model of the drain current of an OTFT is derived. In Section IV, it is compared with the experimental characteristics measured from different transistors, and the physical parameters are discussed. In Section V, conclusions are drawn.

\section{Device Fabrication}

The transistors are fabricated on $25-\mu \mathrm{m}$-thick polyethylene naphthalate (PEN) foil laminated on a removable Si support 

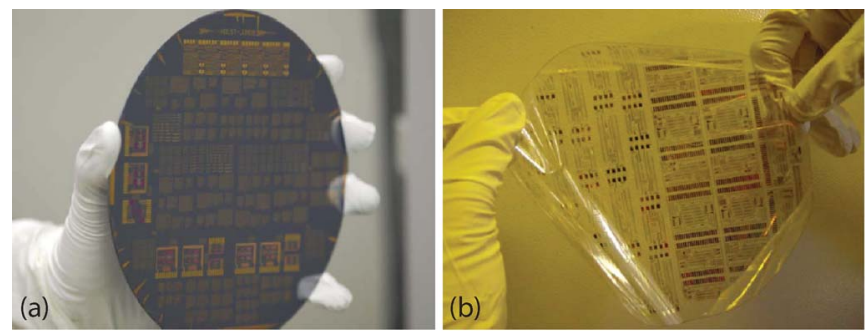

(c)
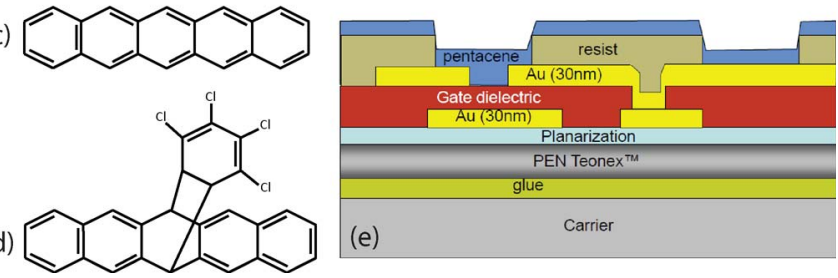

Fig. 1. (a) PEN foil on a rigid silicon wafer. (b) Delaminated foil obtained after the fabrication process. (c) Pentacene molecule. (d) Precursor pentacene molecule. (e) Transistor stack.

wafer of 150-mm diameter (see Fig. 1). Gate electrodes and first-level interconnect lines are made by patterning the gold using standard photolithographic techniques.

Pentacene TFTs: A 100-nm-thick aluminum oxide $\left(\mathrm{Al}_{2} \mathrm{O}_{3}\right)$ is sputtered as a gate dielectric. Source-drain electrodes are gold covered by pentafluorobenzene thiol, which improves hole injection into the p-type semiconductor. The semiconductor is a layer of evaporated pentacene [see Fig. 1(c)], with an average thickness of $30 \mathrm{~nm}$. The transistors were characterized in nitrogen atmosphere using an Agilent 4156C parameter analyzer. The fabrication and measurements have been carried out at IMEC.

Precursor pentacene TFTs: The gate dielectric is a 350-nmthick photoimageable polymer [polyvinyl phenol (PVP)], which is spin coated and subsequently exposed to ultraviolet light to define contact holes. Source-drain electrodes and second-level interconnect lines are defined in the second gold layer. On top of this stack, a 50-nm-thick precursor pentacene [see Fig. 1(d)] film was spin coated. Synthesis of this tetrachlorobenzene precursor and conversion procedure to pentacene are described in [16]. The transistors were fabricated and tested in air, without the use of flow boxes, and using as-purchased materials for the photolithography steps. The fabrication and measurements have been carried out at Polymer Vision.

\section{OTFT MODEL}

Here, the theory proposed in Part I [15] is used to derive the drain current expression of an OTFT. For convenience, we remember that, in the case of a double exponential DOS, the conductivity and the mobility are given by the following equations:

$$
\begin{aligned}
\sigma & =\sigma_{0}\left[\frac{\pi N_{t} T_{t}^{3}}{(2 \alpha)^{3} B_{c} T^{3}}\right]^{\frac{T_{t}}{T}} \exp \left(\frac{\Delta E_{F}}{k_{B} T}\right) \\
\mu & =\left[\frac{1}{\mu_{d}}+\frac{1}{\mu_{t}}\right]^{-1}
\end{aligned}
$$

where $\mu_{d}=\sigma /\left(q n_{d}\right), \mu_{t}=\sigma /\left(q n_{t}\right)$ and $n_{d}, n_{t}$ are the carrier concentrations related to deep and tail states, respectively. $N_{t}$ is the total density of tail states, and $T_{t}$ is the DOS width, which takes into account the energetic disorder of the tail states. $\alpha$ is the charge spatial localization parameter, $B_{c}=2.8, k_{B}$ is the Boltzmann's constant, $T$ is the temperature, and $\Delta E_{F}$ is the energy difference between the Fermi level and the lowest unoccupied molecular orbital (LUMO) one.

In the following, the equations for the drain current are developed for n-channel transistors, and they can be easily rewritten for p-type ones.

The integral expression of the drain current, which is valid in all the regions of operation, can be determined as [6]

$$
I_{D S}=\frac{W}{L} \int_{V_{S}}^{V_{D}} \int_{V_{\mathrm{ch}}}^{\varphi_{s}} \frac{\sigma\left(\varphi, V_{\mathrm{ch}}\right)}{F_{x}\left(\varphi, V_{\mathrm{ch}}\right)} d \varphi d V_{\mathrm{ch}}
$$

where $W$ is the channel width; $L$ is the transistor channel length; $V_{S}$ and $V_{D}$ are the source and drain voltages, respectively; $V_{\mathrm{ch}}$ is the channel potential (i. e. the electron quasi-Fermi level); $\varphi$ is the electrostatic potential; and $\varphi_{s}$ is the electrostatic potential at the gate insulator/organic semiconductor interface. $\sigma\left(\varphi, V_{\mathrm{ch}}\right)$ is calculated according to (1), where $\Delta E_{F}=q\left(\varphi-V_{\mathrm{ch}}\right)+\Delta E_{\mathrm{Fi}}, \Delta E_{\mathrm{Fi}}=E_{\mathrm{Fi}}-E_{\mathrm{LUMO}}$, and $E_{\mathrm{Fi}}=E_{\text {gap }} / 2$. Under the gradual channel approximation, the electric field $F_{x}$ along the direction orthogonal to the insulator/semiconductor surface can be calculated with the 1D Poisson equation, resulting in

$$
F_{x}\left(\varphi, V_{\mathrm{ch}}\right)=\sqrt{\frac{2 q}{\epsilon_{s}} \int_{V_{\mathrm{ch}}}^{\varphi} n\left(\varphi^{\prime}, V_{\mathrm{ch}}\right) d \varphi^{\prime}}
$$

where $\epsilon_{s}=\epsilon_{0} k_{s}, \epsilon_{0}$ is the vacuum permittivity, and $k_{s}$ is the relative permittivity of the semiconductor. In a double exponential DOS, the carrier concentration can be written as $n=n_{d}+n_{t}$, where $n_{d}$ and $n_{t}$ are calculated with [15, eq. (22)] specialized with parameter sets $\left\{N_{d}, T_{d}\right\}$ and $\left\{N_{t}, T_{t}\right\}$, respectively. Substituting the expression of the carrier concentration into (4) and integrating, the drain current can be written as

$$
\begin{aligned}
I_{D S} \simeq \frac{W}{L} \sigma_{0}\left[\frac{\pi N_{t}\left(\frac{T_{t}}{T}\right)^{3}}{(2 \alpha)^{3} B_{c}}\right]^{\frac{T_{t}}{T}} \int_{V_{S}}^{V_{D}} d V_{\mathrm{ch}} \int_{V_{\mathrm{ch}}}^{\varphi_{s}} d \varphi \\
\times \frac{\exp \left[\frac{q\left(\varphi-V_{\mathrm{ch}}\right)+\Delta E_{\mathrm{Fi}}}{k_{B} T}\right]}{\sqrt{\Theta_{d} \exp \left[\frac{q\left(\varphi-V_{\mathrm{ch}}\right)}{k_{B} T_{d}}\right]+\Theta_{t} \exp \left[\frac{q\left(\varphi-V_{\mathrm{ch}}\right)}{k_{B} T_{t}}\right]}}
\end{aligned}
$$

where

$$
\Theta_{o}=N_{o} \frac{2 \pi k_{B} T}{\epsilon_{s} \sin \left(\pi \frac{T}{T_{o}}\right)} \exp \left(\frac{\Delta E_{\mathrm{Fi}}}{k_{B} T_{o}}\right)
$$

and we remember that $o \in\{t, d\}$. In order to solve (5), surface potential $\varphi_{s}$ has to be calculated.

Applying Gauss' law to the insulator/semiconductor interface, the electric field results in a function of the surface potential and the gate voltage: $F_{x}\left(\varphi_{s}\right)=\left(C_{i} / \epsilon_{s}\right)\left(V_{G}-V_{\mathrm{FB}}-\varphi_{s}\right)$, where $C_{i}$ is the gate-insulator capacitance per unit area, and 
$V_{G}$ and $V_{\mathrm{FB}}$ are the gate and flatband voltages, respectively. By equating the latter expression with (4), $\varphi_{s}$ is given by the numerical solution of the following equation:

$$
\sqrt{\frac{2 q}{\epsilon_{s}} \int_{V_{\mathrm{ch}}}^{\varphi_{s}} n\left(\varphi^{\prime}, V_{\mathrm{ch}}\right) d \varphi^{\prime}}=\frac{C_{i}}{\epsilon_{s}}\left(V_{G}-V_{\mathrm{FB}}-\varphi_{s}\right) .
$$

An analytical solution of (5) cannot be found, as the denominator of the integral is the sum of two exponential functions. This mathematical situation has been also encountered in the past for amorphous silicon TFTs [17]-[19]. We would like to highlight that the physics background we assumed in deriving (5) is completely different from that of amorphous silicon, but there are mathematical similarities between the equations. These are summarized here.

1) The VRH conductivity derived in (1) exponentially depends on factor $E_{F} /\left(k_{B} T\right)$. This is the same formulation obtained for a semiconductor where the charge carrier transport takes place in a parabolic band and $\sigma_{\text {band }}=q \mu_{\text {band }} n_{\text {band }}$, band mobility $\mu_{\text {band }}$ is constant, and $n_{\text {band }}=n_{i} \exp \left[E_{F} /\left(k_{B} T\right)\right]$.

2) A double exponential density of traps is assumed.

Following the same approach proposed in [19], the drain current [see (5)] can be rewritten as

$$
\begin{aligned}
I_{D S} & \simeq\left(\frac{1}{I_{\text {deep }}}+\frac{1}{I_{\text {tail }}}\right)^{-1} \\
& =\left(\frac{1}{I_{D}^{\text {Eq. }(5)}\left(\Theta_{t}=0\right)}+\frac{1}{I_{D}^{\text {Eq. }(5)}\left(\Theta_{d}=0\right)}\right)^{-1} .
\end{aligned}
$$

It is interesting to note that the composition rule of the deep and tail currents in (8) is analogous to the one derived for the mobility in (2). This is not surprising and can be interpreted as follows: When the Fermi energy level is located at low energy, hence at low carrier concentration, the mobility is limited by the deep states, and therefore, the drain current is given by the deep-state current. On the contrary, at large concentration, the mobility is limited by the tail states, and the transistor current is defined by the tail-state current. However, we highlight that, from a mathematical point of view, it is not possible to formally derive (8) starting from (2). It is worth noting that (8) is a very good approximation of (5) when the correlation between deep and tail states is low, which means $T_{t} \neq T_{d}$. The accuracy of the approximation increases by increasing the difference between the two characteristic temperatures. Under this hypothesis, the deep and tail states are separately considered, and the overall current can be calculated from the knowledge of the deep and tail currents. This is the key step in order to obtain an analytical expression of the drain current. When a single exponential DOS is considered, the deep and tail currents can be calculated following the same approach proposed in [6]. Thus

$$
\begin{aligned}
I_{\text {deep }} & =\frac{W}{L} \frac{\Xi_{0} \Lambda_{d}}{C_{i}^{2 T_{d} / T}}\left[\left(Q_{\mathrm{aS}}^{\prime}\right)^{\frac{2 T_{d}}{T}}-\left(Q_{\mathrm{aD}}^{\prime}\right)^{\frac{2 T_{d}}{T}}\right] \\
I_{\text {tail }} & =\frac{W}{L} \frac{\Xi_{0} \Lambda_{t}}{C_{i}^{2 T_{t} / T}}\left[\left(Q_{\mathrm{aS}}^{\prime}\right)^{\frac{2 T_{t}}{T}}-\left(Q_{\mathrm{aD}}^{\prime}\right)^{\frac{2 T_{t}}{T}}\right]
\end{aligned}
$$

where

$$
\begin{aligned}
& \Xi_{0}=\sigma_{0} \frac{k_{B} T \epsilon_{s}}{q C_{i}} \\
& \Lambda_{d}=\frac{T}{2 T_{d}-T}\left[\frac{C_{i}^{2} \sin \left(\pi \frac{T}{T_{d}}\right)}{\pi N_{d} 2 k_{B} T \epsilon_{s}}\right]^{\frac{T_{d}}{T}}\left[\frac{\pi N_{t}\left(\frac{T_{t}}{T}\right)^{3}}{(2 \alpha)^{3} B_{c}}\right]^{\frac{T_{t}}{T}} \\
& \Lambda_{t}=\frac{T}{2 T_{t}-T}\left[\frac{C_{i}^{2}\left(\frac{T_{t}}{T}\right)^{4} \sin \left(\pi \frac{T}{T_{t}}\right)}{(2 \alpha)^{3} B_{c} 2 k_{B} T_{t} \epsilon_{s}}\right]^{\frac{T_{t}}{T}}
\end{aligned}
$$

and $Q_{\mathrm{aS}}^{\prime}$ and $Q_{\mathrm{aD}}^{\prime}$ are the accumulated charges per unit area at the source and drain sides of the transistor, respectively.

Finally, substituting into (8)-(10), the overall drain current valid in all the transistor operating regions reads

$$
\begin{aligned}
I_{D S} & =\frac{W}{L} \Xi_{0}\left[\frac{\Lambda_{d} \Lambda_{t} \Phi_{d} \Phi_{t}}{\Lambda_{d} \Phi_{d}+\Lambda_{t} \Phi_{t}}\right] \\
\Phi_{d} & =\left\|V_{G S}-V_{\mathrm{FB}}\right\|^{\frac{2 T_{d}}{T}}-\left\|V_{G S}-V_{\mathrm{FB}}-V_{D S}\right\|^{\frac{2 T_{d}}{T}} \\
\Phi_{t} & =\left\|V_{G S}-V_{\mathrm{FB}}\right\|^{\frac{2 T_{t}}{T}}-\left\|V_{G S}-V_{\mathrm{FB}}-V_{D S}\right\|^{\frac{2 T_{t}}{T}}
\end{aligned}
$$

and operator $\|x\|$ gives $x$ if $x$ is greater than zero, and zero otherwise.

\section{Results AND Discussion}

The modeling results are compared with the experimental data collected from different devices and in a wide range of bias conditions. The derived physical parameters are discussed, and the usefulness of the proposed model is analyzed.

As a first step, we verify that the model based on a single exponential DOS [6] is not suitable to describe our experimental data over the whole biasing range. In order to extract model parameters $T_{o}$, i.e., the DOS characteristic temperature, and flatband voltage $V_{\mathrm{FB}}$ from the measured transfer characteristics, we used the following integral method proposed in [20]:

$$
F\left(V_{S G}\right)=\frac{\int_{0}^{V_{S G}} I_{D S}\left(V_{S G}^{\prime}\right) d V_{S G}^{\prime}}{I_{D S}\left(V_{S G}\right)}
$$

where $F\left(V_{S G}\right)$ is defined according to (15), and it is directly applied on the measured transfer characteristics. This method was successfully applied to amorphous silicon and organic TFTs under the assumption that the mobility follows power law dependence on the gate voltage (this condition is fulfilled when an exponential DOS is assumed). An analytical expression of (15) can be obtained by separately considering the linear and saturation regions of the transistor. In the linear region, the drain current has to be approximated with a first-order Taylor expansion, and substituting the mathematical expression into (15) results in

$$
F_{\mathrm{Lin}}\left(V_{S G}\right) \simeq \frac{T}{2 T_{o}}\left(V_{S G}-V_{\mathrm{FB}}\right)
$$

In the saturation region, the current can be directly substituted into (15) without approximations and gives

$$
F_{\mathrm{Sat}}\left(V_{S G}\right)=\frac{T}{2 T_{o}+T}\left(V_{S G}-V_{\mathrm{FB}}\right) .
$$




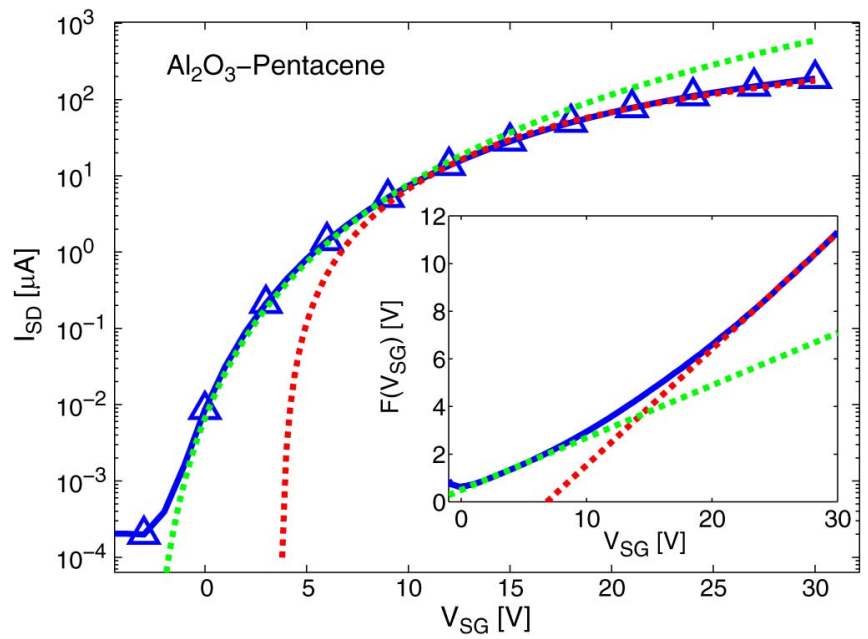

Fig. 2. (Main panel) Transfer characteristics at $V_{S D}=10 \mathrm{~V}$. The blue line with symbols shows the measured data, whereas the dashed red and green lines are the fits with a single exponential DOS model [6]. (Inset) The blue full line is the functional $F\left(V_{S G}\right)$ calculated according to (15), whereas the dashed red and green lines are obtained fitting (16) and (17) at large and small $V_{S G}$, respectively.

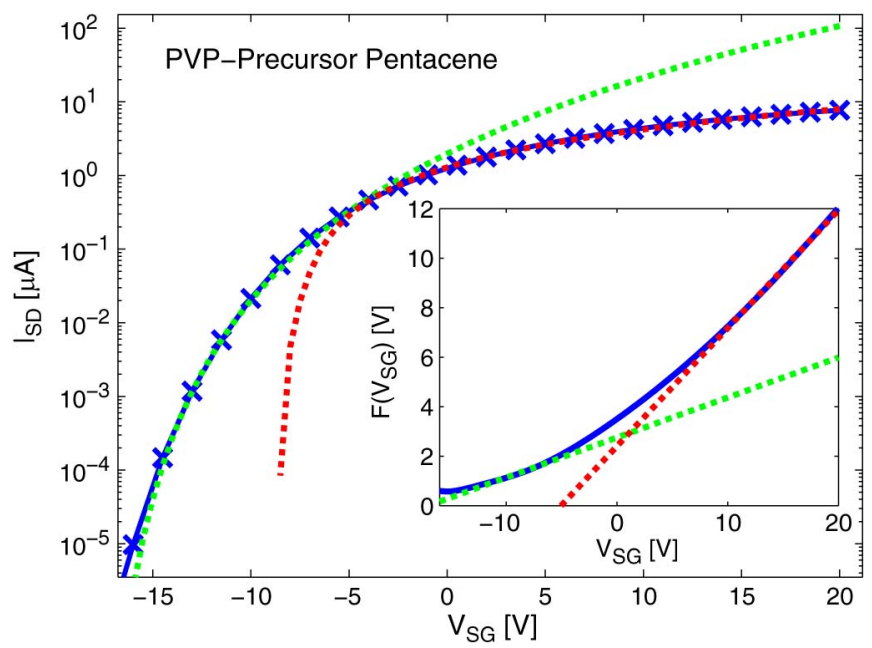

Fig. 3. (Main panel) Transfer characteristics at $V_{S D}=1 \mathrm{~V}$. The blue line with symbols shows the measured data, whereas the dashed green and red lines are the fits with a single exponential DOS model [6]. (Inset) The blue full line is the functional $F\left(V_{S G}\right)$ calculated according to (15), whereas the dashed red and green lines are obtained fitting (16) at small and large $V_{S G}$, respectively.

By applying function (15) to the measured transfer characteristics of a transistor operating in the linear or saturation region, the numerical quantity $F\left(V_{S G}\right)$ is obtained, and it can be fitted with (16) or (17). This procedure yields physical parameters $T_{o}$ and $V_{\mathrm{FB}}$.

The results of this method are plotted in the inset in Figs. 2 and 3 . The blue line is the quantity $F\left(V_{S G}\right)$ obtained from experimental data: it cannot be fitted by a straight line [see (16) and (17)] in the whole range of gate potentials. Furthermore, the shape of $F\left(V_{S G}\right)$ also suggests that it could be approximated by two straight lines: one for high values of $V_{S G}$ (dashed red line) and the other for low $V_{S G}$ (dashed green line).

By fitting the single exponential model on these two intervals, the corresponding drain currents are compared with the measurements in the main panel in Figs. 2 and 3. Both transistors show the same results. At small values of $V_{S G}$,
TABLE I

Technological, Geometrical, and Physical Parameters of the TRANSISTORS. $W$ : TRANSISTOR WIDTH; $L$ : TRANSISTOR CHANNEL LengTh; $k_{\text {osc }}$ : PentaCene Dielectric Constant (VAlue TakeN From [8]); $C_{i}$ : Gate-InSUlator Capacitance PER Unit AREA; $\mu_{\mathrm{FE}}=\left(L /\left(W C_{i} V_{S D}\right)\right) \times\left(\partial I_{S D} / \partial V_{S G}\right):$ LINEAR FIELD-EFFECT MOBILITY CALCULATED AT $V_{S G}-V_{\mathrm{FB}}=20 \mathrm{~V} ; \alpha^{-1}$ : LOCALIZATION Radius (Value Taken From [8]); $T$ : Device Temperature; $\sigma_{0}$ : Conductivity Prefactor; $N_{t}$ : TOTAL Number OF TAIL States (Typical Value, TAKen From [23]); $N_{d}$ : TOtal Number of DeEP States; $T_{t}$ : TAIL-STATE CharaCTERISTIC TEMPERATURE; $T_{d}$ : DeEP-STATE CharaCteristiC Temperature; $V_{\mathrm{FB}}$ : Flatband Voltage

\begin{tabular}{|c|c|c|}
\hline & OTFT 1 (T1) & OTFT 2 (T2) \\
\hline Organic SemiConductor & Pentacene & Precursor Pentacene \\
\hline OSC deposition & Evaporation & Spincoating \\
\hline$W[\mu m]$ & 1000 & 500 \\
\hline$L[\mu \mathrm{m}]$ & 10 & 5 \\
\hline$k_{\text {OSC }}$ & 3 & 3 \\
\hline Gate insulator & $\mathrm{Al}_{2} \mathrm{O}_{3}$ & $P V P$ \\
\hline$C_{i}\left[\mathrm{nF} / \mathrm{cm}^{2}\right]$ & 70 & 9.5 \\
\hline$\mu_{F E}\left[\mathrm{~cm}^{2} / V s\right]$ & 0.045 & 0.154 \\
\hline$\alpha^{-1}[\AA]$ & 2.2 & 2.2 \\
\hline$T[K]$ & 298 & 298 \\
\hline$\sigma_{0}[S / \mathrm{cm}]$ & $1 \times 10^{5}$ & $4.65 \times 10^{5}$ \\
\hline$N_{t}\left[\mathrm{~cm}^{-3}\right]$ & $1 \times 10^{21}$ & $1 \times 10^{21}$ \\
\hline$N_{d}\left[\mathrm{~cm}^{-3}\right]$ & $4.5 \times 10^{20}$ & $3.6 \times 10^{19}$ \\
\hline$T_{t}[K]$ & 360 & 360 \\
\hline$T_{d}[K]$ & 850 & 907 \\
\hline$V_{F B}[V]$ & 4.3 & 17.7 \\
\hline
\end{tabular}

corresponding to low energies in the DOS, the transistor works in weak accumulation (subthreshold region), and the deepstate exponential DOS accurately reproduces the experimental behavior. By increasing $V_{S G}$, the Fermi level shifts to higher energies, and in strong accumulation, the current is overestimated by the deep-state current. On the other hand, in this operating region, the current calculated by considering only the tail states accurately reproduces the measurements.

This analysis clearly shows that the actual DOS of our devices is well described with a double exponential function, and hence, the theory presented in Part I [15] can be used to model the transistors. The fitting procedure gives a total of six parameters, namely, $\left\{\sigma_{0 d}, T_{0 d}, V_{\mathrm{FBd}}\right\}$ and $\left\{\sigma_{0 t}, T_{0 t}, V_{\mathrm{FBt}}\right\}$. The first set is obtained from the fit at low $V_{S G}$, and it is used to calculate the deep-state current, whereas the second parameters set is for the tail-state current. It is worth noting that $V_{\mathrm{FBt}}$ has to be considered as an equivalent flatband voltage, which takes into account the global effect of the charge localized in the deep states.

These results can be unified in a physically consistent formulation due to the proposed theory. Since the transistor conductivity only depends on the tail-state distribution [see (1)], in strong accumulation, the current is only defined by the tail exponential and results in $\sigma_{0}=\sigma_{0 t}$ and $T_{t}=T_{0 t}$. When the transistor works in weak accumulation, the deep-state distribution dominates the current, and from this operating region, we have $V_{\mathrm{FB}}=V_{\mathrm{FBd}}, T_{d}=T_{0 d}$, and $N_{d} \propto\left(N_{t}\right)^{T_{t} / T_{d}} /\left(\sigma_{0 d}\right)^{T / T_{d}}$. Since the model only depends on ratio $N_{t} / N_{d}$, we chose the same value of total number of tail states for the two semiconductors: $N_{t}=10^{21} \mathrm{~cm}^{-3}$ [23]. Values of $N_{t}$ between $10^{20}$ and $10^{22} \mathrm{~cm}^{-3}$ are usually assumed for organic semiconductors [21]-[23]. The numerical values of parameters obtained for the different OTFTs are reported in Table I. It is important to 


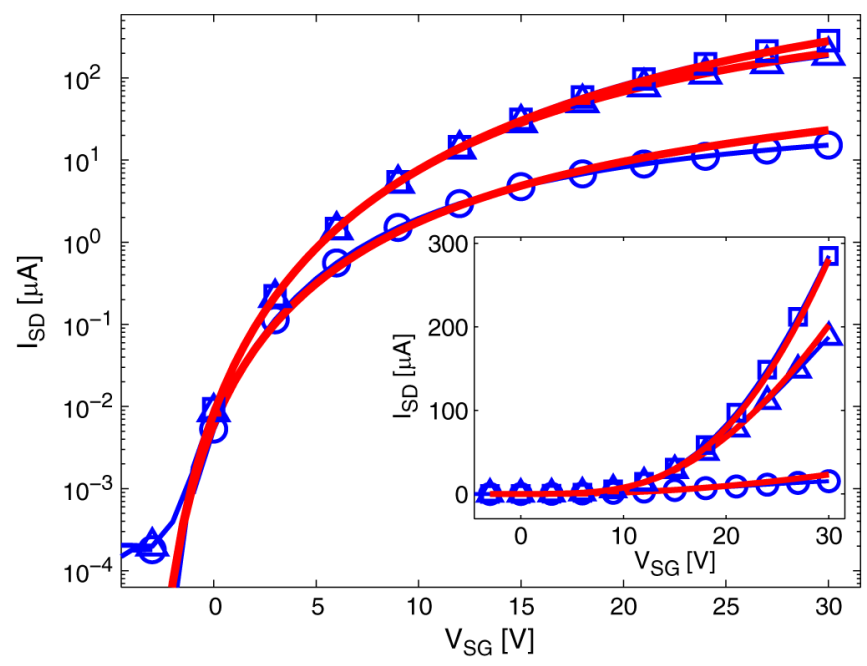

Fig. 4. (Blue symbols and lines) Measured and (red lines) modeled transfer characteristics of an $\mathrm{Al}_{2} \mathrm{O}_{3}$-pentacene TFT for several source-drain voltages. (○) $V_{S D}=1 \mathrm{~V}$. $(\triangle) V_{S D}=10 \mathrm{~V}$. ( $\left.\square\right) V_{S D}=20 \mathrm{~V}$. The geometrical and physical parameters of the transistor model [see (14)] are reported in Table I.

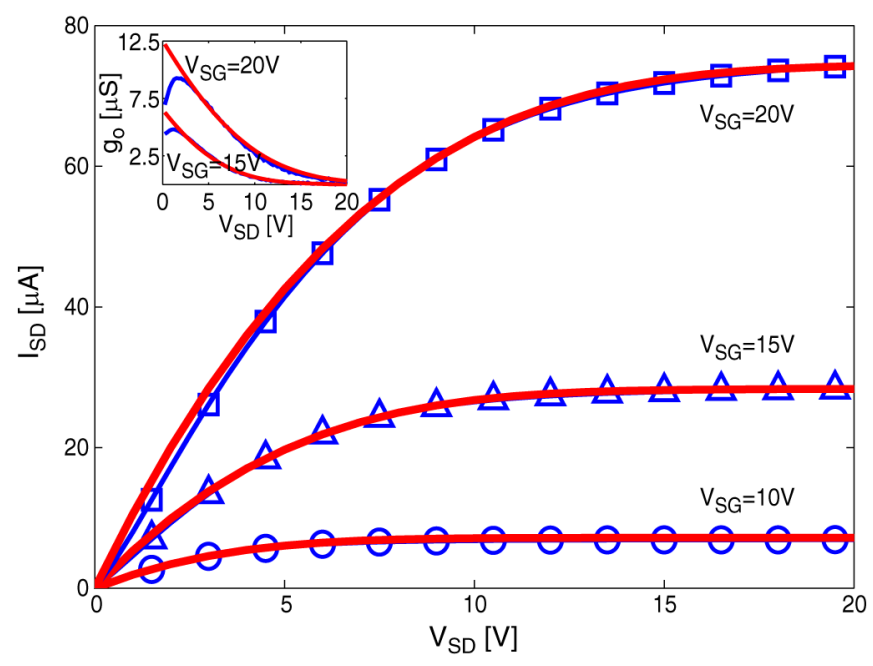

Fig. 5. (Main panel) Output characteristics and (inset) output conductance values of an $\mathrm{Al}_{2} \mathrm{O}_{3}$-pentacene TFT. The blue symbols and lines are the experimental data, whereas the red lines are obtained with the analytical model of (14).

note that the hypothesis behind the theory are fulfilled both in transistors $\mathrm{T} 1$ (pentacene) and $\mathrm{T} 2$ (precursor pentacene) since $\chi \equiv T_{d} / T_{t}$ results in $\chi_{T 1}=2.36$ and $\chi_{T 2}=2.52$, and $\eta \equiv$ $N_{d} / N_{t}$ results in $\eta_{T 1}=0.45$ and $\eta_{T 2}=0.036$. The values of $\eta$ and $\chi$ obtained from both transistors fully justify approximation $E_{b} \simeq 0$, and hence, the conductivity only depends on the tail states (see [15, Sec. II and Fig. 2]).

The comparisons between the measurements and the analytical model [see (14)] are shown in Figs. 4-6 for $\mathrm{Al}_{2} \mathrm{O}_{3}-$ pentacene and PVP-precursor pentacene TFTs. There is a very good agreement between the experimental data and the model for both devices in the whole range of biasing conditions. In the case of a pentacene TFT (see Fig. 4), the model slightly overestimates the current at large gate voltages and small $V_{S D}$. From the shape of the output conductance drawn in the inset in Fig. 5, we can see that it is due to contact effects. Their con-

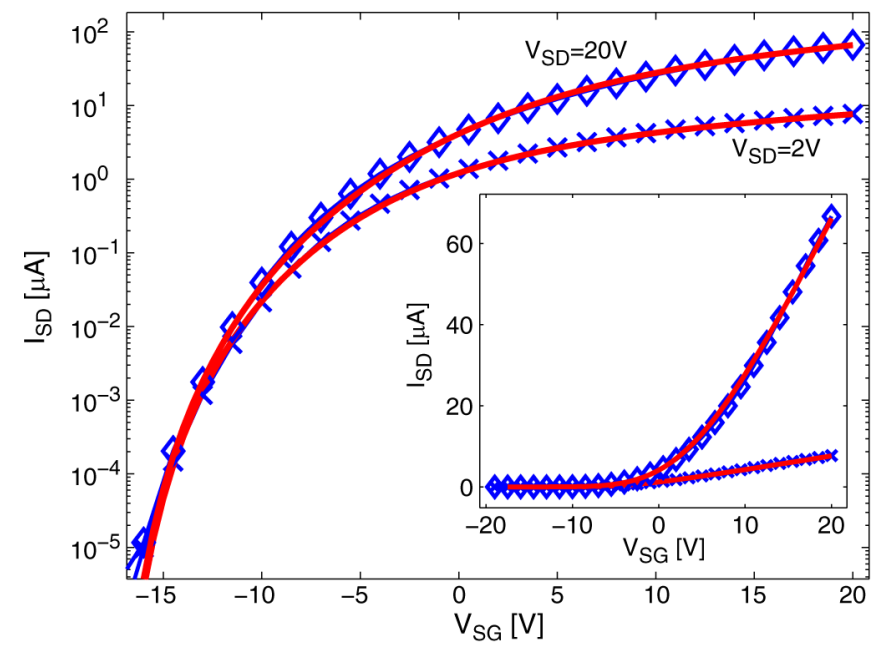

Fig. 6. (Blue symbols and lines) Measured and (red lines) modeled transfer characteristics of a PVP-precursor pentacene TFT. The geometrical and physical parameters of the transistor are reported in Table I.

tributions are not included in the presented model and will be treated in future work. Although in the case we are considering the contact resistances are a minor effect, they may lead to a wrong estimation of the tail-state parameters. In such situation, the extraction procedure [see (15) and (17)] has to be applied on the transfer characteristic measured at higher drain voltages. The detailed discussion on the parameter extraction procedures is beyond the scope of this paper. Further considerations can be found in [24] for a single exponential DOS, and they can be extended to the case of a double exponential function as discussed above.

Finally, in the light of the theory and the analytical model, physical information on the materials used to fabricate the transistors can be derived. 1) From the comparison of $\sigma_{0}$ results that the channel conductivity of precursor pentacene deposited on a PVP insulator is about four times the conductivity of pentacene on $\mathrm{Al}_{2} \mathrm{O}_{3}$, and this is confirmed by the estimated field-effect mobility $\mu_{\mathrm{FE}}$ reported in Table I. 2) The total number of deep states in precursor pentacene is one order of magnitude less than the value of $N_{d}$ obtained for pentacene, and the DOS width is slightly broader, i.e., $T_{d_{T 2}}>T_{d_{T 1}}$. 3) The two semiconductors have the same tail-state temperature.

These results can be interpreted as follows. Tanase et al. [21] showed that the tail-state distribution extracted from organic light-emitting diodes can be unified with the DOS extracted from OTFTs made with the same organic materials. On the other hand, Ke et al. [25] showed that the distribution of interface traps and deep states strongly depends on the gateinsulator properties. Moreover, the experimental study provided by Veres et al. [26] indicates that the gate insulator changes the DOS of the organic semiconductor.

These findings suggest that the tail states are an intrinsic property of the organic semiconductor, whereas the deep states characterize the interaction between the semiconductor and the gate insulator. Therefore, by comparing the extracted parameters, we should conclude that precursor pentacene has been fully converted in pentacene, the two fabrication processes give semiconductors with the same intrinsic disorder $\left(T_{t_{T 1}}=T_{t_{T 2}}\right)$, 


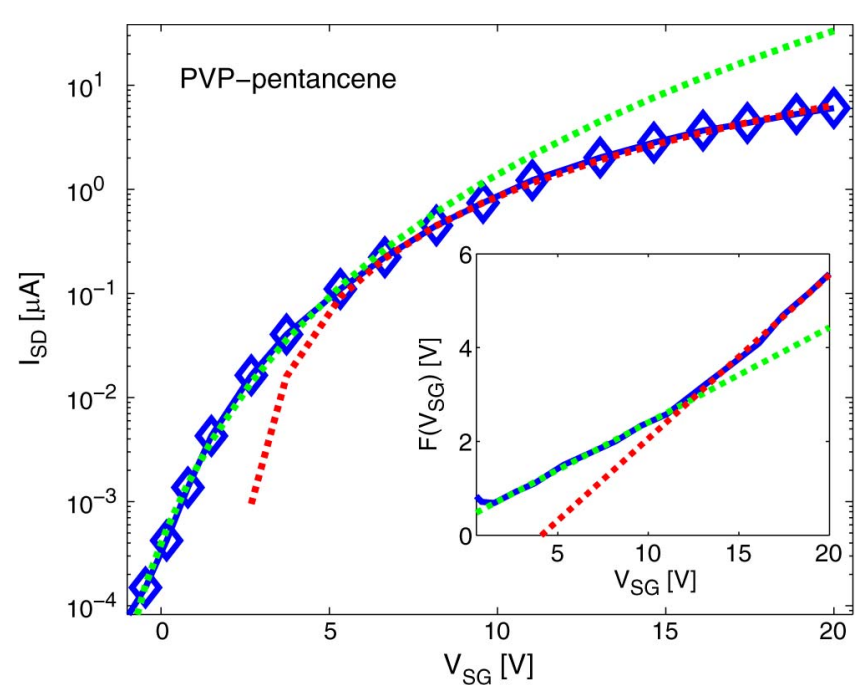

Fig. 7. (Main panel) Transfer characteristics at $V_{S D}=20 \mathrm{~V}$. The blue line with symbols shows the experimental data taken from [27], whereas the dashed red and green lines are the fits with a single exponential DOS model [6]. (Inset) The blue full line is the functional $F\left(V_{S G}\right)$ calculated according to (15), whereas the dashed green and red lines are obtained fitting (17) at small and large $V_{S G}$, respectively.

and the interface PVP-pentacene has less traps with respect to the $\mathrm{Al}_{2} \mathrm{O}_{3} /$ pentacene one $\left(N_{d_{T 2}} \ll N_{d_{T 1}}\right)$. This indicates that pentacene is well formed on the PVP dielectric. This analysis show that the disorder of the organic semiconductor is enhanced by the insulator with large permittivities, and it is in agreement with the study presented in [26].

In order to investigate more in detail the nature of the tail and deep trap states, we model other four OTFTs [27], which are made with the same organic active layer (evaporated pentacene) but have different gate-insulator materials: PVP, PVP copolymer, $\mathrm{SiO}_{2}$ treated with OTS, and $\mathrm{SiO}_{2}$.

Following the same procedure described before, for each OTFT $(T 3-T 6)$, the functional $F\left(V_{S G}\right)$ is calculated by applying (15) to the transfer characteristic, and then, it is fitted by means of (17). The results are displayed in the inset in Figs. 7 and 8 for transistors made with PVP-pentancene (T3) and $\mathrm{SiO}_{2}+$ OTS-pentancene (T5), respectively. As shown in the inset in Fig. 7, in the case of $T 3$, the quantity $F\left(V_{S G}\right)$ obtained from the experimental data cannot be fitted by a straight line in the whole range of $V_{S G}$. According to the previous analysis, the DOS can be accurately approximated with a double exponential function, and the proposed model [see (14)] is needed to describe the OTFT in all the regions of operation. On the other hand, the functional calculated for $T 5$ (inset in Fig. 8) can be fitted by means of only one straight line, and hence, a single exponential DOS is accurate enough to model the OTFT with the $\mathrm{SiO}_{2}+$ OTS dielectric. This result is in agreement with other works [8], [28], which successfully modeled the mobility of OTFTs by means of a single exponential DOS.

Transistors PVP copolymer-pentancene (T4) and $\mathrm{SiO}_{2}-$ pentancene (T6) behave as $T 3$ and $T 5$, respectively. The fitted physical parameters of transistors $T 3-T 6$ are reported in Table II, and the comparisons between the measurements and the model are displayed in the four panels in Fig. 9. It is worth to note that the DOS parameters obtained for $T 3$ (PVP) and $T 4$

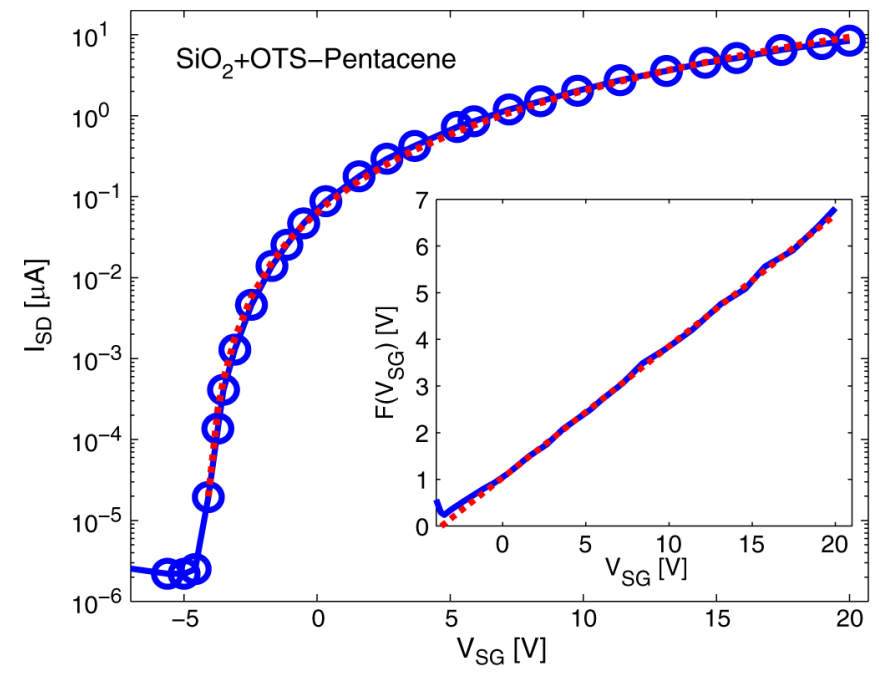

Fig. 8. (Main panel) Transfer characteristics at $V_{S D}=20 \mathrm{~V}$. The blue line with symbols shows the experimental data taken from [27], whereas the dashed red line is fitted with a single exponential DOS model [6]. (Inset) The blue full line is the functional $F\left(V_{S G}\right)$ calculated according to (15), whereas the dashed red line is fitted using (17).

(PVP cop.) are in very good agreement with the ones calculated for $T 2$ (PVP).

These results greatly support that the tail states are an intrinsic property of the organic semiconductor, whereas the deep states arise from the interaction between the semiconductor and the gate insulator. When the gate insulator introduces additional deep-energy traps in the semiconductor, the resulting DOS can be approximated by a double exponential function, and the proposed model can be used to accurately describe the drain current of the OTFTs.

Finally, it is worth to note that the functional $F\left(V_{S G}\right)$ gives information about the shape of the DOS; hence, it is a useful tool to understand when the double exponential model is needed, and it can be used to accomplish an a priori choice. In fact, after the calculation of $F\left(V_{S G}\right)$ directly from a single transfer characteristic, the OTFT model based on a single exponential DOS [6] or on a double exponential DOS [see (14)] can be appropriately chosen.

\section{CONCLUSiON}

An analytical model for the drain current of OTFTs is derived. This extends the work in [6] to the case of a double exponential DOS. The model has been validated with experimental data collected from transistors made with different semiconductors, fabrication processes, and gate insulators.

The measurements have been interpreted by means of the proposed theory and model. The tail states are an intrinsic property of the organic semiconductor, whereas the deep states are induced by the gate insulator. When the interaction between the gate insulator and the organic semiconductor is weak, the DOS is composed by only tail states that can be approximated with a single exponential function. This is in agreement with other works [4]-[6], [8], [28]. On the other hand, when the gate insulator introduces additional traps in the semiconductor, the overall DOS can be approximated with a double exponential 
TABLE II

Technological, Geometrical, and Physical Parameters of the Transistors. $W$ : Transistor Width; $L:$ Transistor Channel Length; $k_{\text {osc }}$ : Pentacene Dielectric Constant (Value Taken From [8]);

$C_{i}$ : Gate-Insulator Capacitance Per Unit Area (VAlues Taken From [27]); $\alpha^{-1}$ : Localization Radius (Value Taken From [8]); $T$ : Device Temperature; $\sigma_{0}$ : Conductivity Prefactor; $N_{t}$ : Total Number of Tail States (Typical Value, Taken From [23]); $N_{d}$ : Total Number OF DeEP States; $T_{t}$ : TAil-State Characteristic TeMPerature; $T_{d}$ : DeEP-STATE Characteristic Temperature; $V_{\mathrm{FB}}$ : Flatband Voltage

\begin{tabular}{|l||c|c|c|c|}
\hline & OTFT 3 (T3) & OTFT 4 (T4) & OTFT 5 (T5) & OTFT 6 (T6) \\
\hline Organic SemiC. & Pentacene & Pentacene & Pentacene & Pentacene \\
\hline OSC deposition & Evaporation & Evaporation & Evaporation & Evaporation \\
\hline$W[\mu \mathrm{m}]$ & 170 & 170 & 170 & 170 \\
\hline$L[\mu \mathrm{m}]$ & 130 & 130 & 130 & 130 \\
\hline$k_{\text {Osc }}$ & 3 & 3 & 3 & 3 \\
\hline Gate insulator & PVP & PVP cop. & SiO $_{2}+$ OTS & $\mathrm{SiO}_{2}$ \\
\hline$C_{i}\left[\mathrm{nF} / \mathrm{cm}^{2}\right]$ & 12.26 & 9.32 & 34.53 & 34.53 \\
\hline$\alpha^{-1}[\AA]$ & 2.2 & 2.2 & 2.2 & 2.2 \\
\hline$T[\mathrm{~K}]$ & 295 & 295 & 295 & 295 \\
\hline$\sigma_{0}\left[\mathrm{~S} / \mathrm{cm}^{-3}\right]$ & $9.3 \times 10^{6}$ & $18.6 \times 10^{6}$ & $6 \times 10^{6}$ & $3.2 \times 10^{6}$ \\
\hline$N_{t}\left[\mathrm{~cm}{ }^{-3}\right]$ & $1 \times 10^{21}$ & $1 \times 10^{21}$ & $1 \times 10^{21}$ & $1 \times 10^{21}$ \\
\hline$N_{d}\left[\mathrm{~cm}{ }^{-3}\right]$ & $3.6 \times 10^{19}$ & $3.6 \times 10^{19}$ & - & - \\
\hline$T_{t}[\mathrm{~K}]$ & 360 & 360 & 426.6 & 494 \\
\hline$T_{d}[\mathrm{~K}]$ & 820 & 855 & - & - \\
\hline$V_{F B}[\mathrm{~V}]$ & 3 & 2.5 & 4.35 & 11.2 \\
\hline
\end{tabular}

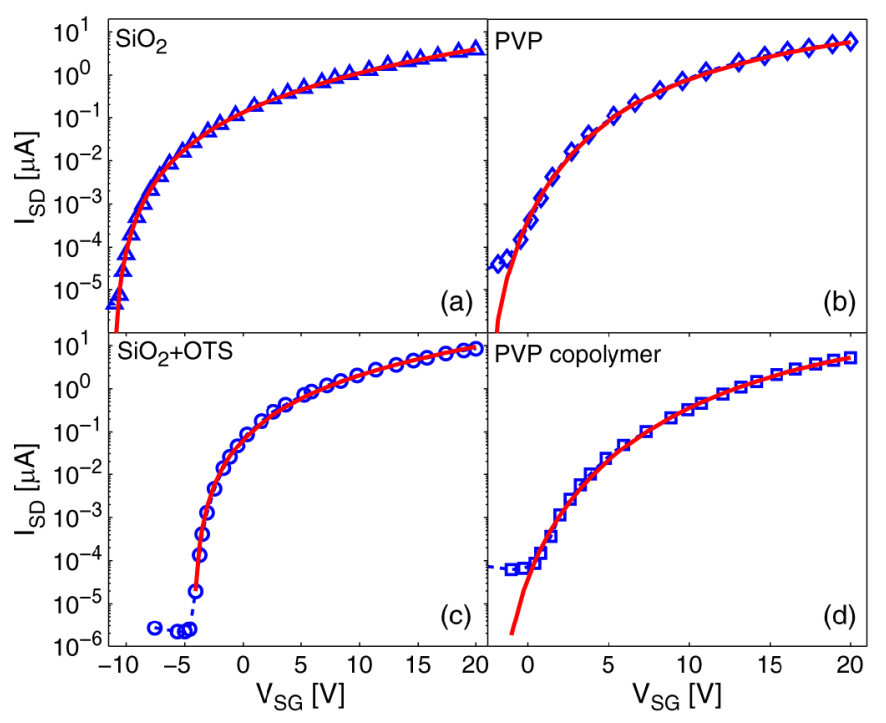

Fig. 9. (Blue dashed lines with symbols) Measured and (red full lines) modeled transfer characteristics of pentacene TFTs with different gate insulator materials. Experimental data are taken from [27]. The geometrical and physical parameters of the transistors are reported in Table II. $V_{S D}=20 \mathrm{~V}$.

function, and this is in agreement with many studies presented in the literature [9]-[13].

In the case of a double exponential DOS, when the OTFTs work in weak accumulation (subthreshold region), the current is dominated by the charge carrier transport in the deep states of the DOS, whereas when the transistors are biased in the linear region, the channel is strongly accumulated, and the current is defined by the tail states.

The proposed analytical OTFT model is of general applicability and can be further extended in order to account for the channel modulation and contact effects. This physically based model can be thus considered as the basic building block of a more general framework useful to investigate the physics of organic semiconductors and for circuit simulation.

\section{REFERENCES}

[1] G. Horowitz and P. Delannoy, "An analytical model for organic-based thin-film transistors," J. Appl. Phys., vol. 70, no. 1, pp. 469-475, Jul. 1991.

[2] P. V. Necliudov, M. S. Shur, D. J. Gundlach, and T. N. Jackson, "Modeling of organic thin film transistors of different designs," J. Appl. Phys., vol. 88, no. 11, pp. 6594-6597, Dec. 2000.

[3] M. Estrada, A. Cerdeira, J. Puigdollers, L. Reséndiz, J. Pallares, L. F. Marsal, C. Voz, and B. Iñiguez, "Accurate modeling and parameter extraction method for organic TFTs," Solid State Electron., vol. 49, no. 6, pp. 1009-1016, Jun. 2005.

[4] E. Calvetti, L. Colalongo, and Zs. M. Kovacs-Vajna, "Organic thin film transistors: A DC/dynamic model for circuit simulation," Solid State Electron., vol. 49, no. 4, pp. 567-577, Apr. 2005.

[5] M. Fadlallah, W. Benzarti, G. Billiot, W. Eccleston, and D. Barclay, "Modeling and characterization of organic thin film transistors for circuit design," J. Appl. Phys., vol. 99, no. 10, p. 104 504, May 2006.

[6] F. Torricelli, Z. M. Kovács-Vajna, and L. Colalongo, "A charge-based OTFT model for circuit simulation," IEEE Trans. Electron Devices, vol. 56, no. 1, pp. 20-30, Jan. 2009.

[7] O. Marinov, M. J. Deen, U. Zschieschang, and H. Klauk, "Organic thinfilm transistors: Part I-Compact DC modeling," IEEE Trans. Electron Devices, vol. 56, no. 12, pp. 2952-2961, Dec. 2009.

[8] M. C. J. M. Vissenberg and M. Matters, "Theory of the field-effect mobility in amorphous organic transistors," Phys. Rev. B, Condens. Matter Mater. Phys., vol. 57, no. 20, pp. 12964-12 967, May 1998.

[9] O. Tal, Y. Rosenwaks, Y. Preezant, N. Tessler, C. K. Chan, and A. Kahn, "Direct determination of the hole density of states in undoped and doped amorphous organic films with high lateral resolution," Phys. Rev. Lett., vol. 95, no. 25, pp. 256 405/1-256 405/4, Dec. 2005.

[10] D. V. Lang, X. Chi, T. Siegrist, A. M. Sergent, and A. P. Ramirez, "Amorphous like density of gap states in single-crystal pentacene," Phys. Rev. Lett., vol. 93, no. 8, pp. 086 802/1-086 802/4, Aug. 2004.

[11] J. Puigdollers, A. Marsal, S. Cheylan, C. Voz, and R. Alcubilla, "Densityof-states in pentacene from the electrical characteristics of thin-film transistors," Organic Electron., vol. 11, no. 8, pp. 1333-1337, Aug. 2010.

[12] J. Puigdollers, M. Della Pirriera, A. Marsal, A. Orpella, S. Cheylan, C. Voz, and R. Alcubilla, "N-type PTCDI- $\mathrm{C}_{13} \mathrm{H}_{27}$ thin-film transistors deposited at different substrate temperature," Thin Solid Films, vol. 517, no. 23 , pp. 6271-6274, Oct. 2009.

[13] F. De Angelis, L. Mariucci, S. Cipolloni, and G. Fortunato, "Analysis of electrical characteristics of high performance pentacene thin-film transistors with PMMA buffer layer," J. Non-Cryst. Solids, vol. 352, no. 9-20, pp. 1765-1768, Jun. 2006.

[14] N. Hulea, H. B. Brom, A. J. Houtepen, D. Vanmaekelbergh, J. J. Kelly, and E. A. Meulenkamp, "Wide energy-window view on the density of states and hole mobility in poly(p-phenylene vinylene)," Phys. Rev. Lett., vol. 93, no. 16, pp. 166 601/1-166601/3, Oct. 2004. 
[15] F. Torricelli, "Charge transport in organic transistors accounting for a wide distribution of carrier energies-Part I: Theory," IEEE Trans. Electron Devices, to be published.

[16] P. T. Herwig and K. Müllen, "A soluble pentacene precursor: Synthesis, solid-state conversion into pentacene and application in a field-effect transistor," Adv. Mater., vol. 11, no. 6, pp. 480-483, Apr. 1999.

[17] K. Khakzar and E. H. Lueder, "Modeling of amorphous-silicon thin-film transistors for circuit simulations with SPICE," IEEE Trans. Electron Devices, vol. 39, no. 6, pp. 1428-1434, Jun. 1992.

[18] S. Chen and J. B. Kuo, "An analytical a-Si:H TFT DC/capacitance model using an effective temperature approach for deriving a switching time model for an inverter circuit considering deep and tail states," IEEE Trans. Electron Devices, vol. 41, no. 7, pp. 1169-1178, Jul. 1994.

[19] L. Colalongo, "A new analytical model for amorphous-silicon thin-film transistor including tail and deep states," Solid State Electron, vol. 45, no. 9, pp. 1525-1530, Sep. 2001.

[20] A. Cerdeira, M. Estrada, R. García, A. Ortiz-Conde, and F. J. García Sánchez, "New procedure for the extraction of basic a-Si:H TFT model parameters in the linear and saturation regions," Solid State Electron, vol. 45, no. 7, pp. 1077-1080, Jul. 2001.

[21] C. Tanase, E. J. Meijer, P. W. M. Blom, and D. M. de Leeuw, "Unification of the hole transport in polymeric field-effect transistors and light-emitting diodes," Phys. Rev. Lett., vol. 91, no. 21, pp. 216 601-216604, Nov. 2003.

[22] F. Torricelli, Z. M. Kovács-Vajna, and L. Colalongo, "The role of the density of states on the hole mobility of disordered organic semiconductors," Organic Electron., vol. 10, no. 5, pp. 1037-1040, Aug. 2009.

[23] S. Scheinert and G. Paasch, "Interdependence of contact properties and field- and density-dependent mobility in organic field-effect transistors," J. Appl. Phys., vol. 105, no. 1, pp. 014 509/1-014 509/9, Jan. 2009.

[24] M. J. Deen, O. Marinov, U. Zschieschang, and H. Klauk, "Organic thinfilm transistors: Part II-Parameter extraction," IEEE Trans. Electron Devices, vol. 56, no. 12, pp. 2962-2968, Dec. 2009

[25] L. Ke, S. B. Dolmanan, C. Vijila, S. J. Chua, Y. H. Han, and T. Mei, "Investigation of the device degradation mechanism in pentacene-based thinfilm transistors using low-frequency-noise spectroscopy," IEEE Trans. Electron Devices, vol. 57, no. 2, pp. 385-390, Feb. 2010.

[26] J. Veres, S. D. Ogier, S. W. Leeming, D. C. Cupertino, and S. M. Khaffaf, "Low- $k$ insulators as the choice of dielectric in organic field-effect transistors," Adv. Funct. Mater, vol. 13, no. 3, pp. 199-204, Mar. 2003.

[27] H. Klauk, M. Halik, U. Zschieschang, G. Schmid, W. Radlik, and W. Weber, "High-mobility polymer gate dielectric pentacene thin film transistors," J. Appl. Phys., vol. 92, no. 9, pp. 5259-5263, Nov. 2002.

[28] M. Estrada, A. Cerdeira, I. Meija, M. Avila, R. Picos, L. F. Marsal, J. Pallares, and B. Iñiguez, "Modeling the behavior of charge carrier mobility with temperature in thin-film polymeric transistors," Microelectron. Eng., vol. 87 , no. 12 , pp. 2565-2570, Dec. 2010.

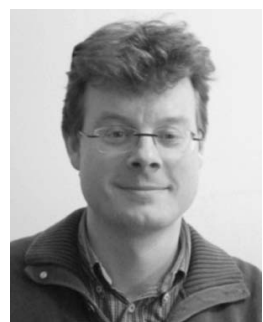

Kevin O'Neill was born in the United States in 1974. He received the B.A. (hons.) degree in natural sciences (physics) from the University of Oxford, Oxford, U.K., in 1996, the M.Sc. degree (with distinction) in semiconductor science and technology from the Imperial College of Science, Technology and Medicine, University of London, London, U.K., in 1997, and the M.Sc. and Ph.D. degrees in physics from Cornell University, Ithaca NY, in 2000 and 2003, respectively.

From 2003 to 2007, he was a Postdoctoral Researcher with the Technical University of Delft, first as a European Union Marie Curie Fellow and then as a Dutch Government Veni Fellow. From 2007 to 2011, he was a Senior Scientist with the Research and Development section of Polymer Vision, BV. Since 2011, he has been a Principal Design Engineer with SensL Technologies, Ltd., Cork, Ireland. His research interests include optical properties of multiquantum-well systems, charge transport in low-dimensional metals, single-molecule electronic devices, integration and design of organic and oxide thin-film transistors for large area displays, and, most recently, the design of avalanche photo detectors and silicon photomultipliers for low-lightlevel detection devices.

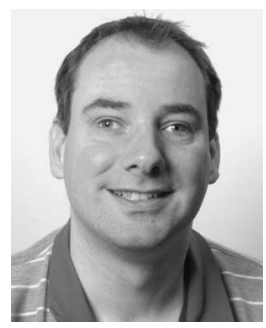

Gerwin H. Gelinck received the Ph.D. degree from Delft University of Technology, Delft, The Netherlands, in 1998 .

In 1998, he joined as a Senior Scientist with Philips Research, where he started working on polymer and organic transistors and their use in integrated circuits, displays, and memory devices. In 2002, he was a cofounder of Polymer Vision, Eindhoven, The Netherlands. From 2002 to 2006, he was a Chief Scientist with Polymer Vision. Since 2007, he has been a Program Manager of "Organic and Oxide Circuitry" with Holst Centre (a joint research initiative of TNO and IMEC), Eindhoven.

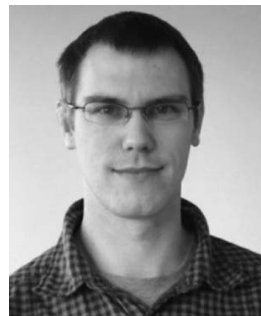

Kris Myny (S'08) was born in Hasselt, Belgium, on July 26, 1980. He received the Master's degree from the Katholieke Hogeschool Limburg, Diepenbeek, Belgium, in 2002. He is currently working toward the $\mathrm{Ph} . \mathrm{D}$. degree in the design of organic circuits at the Katholieke Universiteit Leuven, Leuven, Belgium.

In 2004, he joined IMEC, Leuven, as a member of the Polymer and Molecular Electronics Group. His main research interests are the design, fabrication, and optimization of digital organic circuits for, among others, organic RFID tags and AMOLED backplanes.

Mr. Myny was the recipient of the IMEC 2010 Scientific Excellence Award and the 2011-2012 IEEE SSCS Predoctoral Achievement Award.

Jan Genoe (S'87-M'02) was born in Leuven, Belgium, on May 19, 1965. He received the M.S. degree in electrical engineering and the Ph.D. degree from the Katholieke Universiteit Leuven, Leuven, Belgium, in 1988 and 1994, respectively.

In 1994, he joined the Grenoble High Magnetic Field Laboratory as a Human Capital and Mobility Fellow of the European Community. In 1997, he became a Lecturer with the Katholieke Hogeschool Limburg (KHLim), Diepenbeek, Belgium. Since 2003, he has been both a Professor with KHLim and the Head of the Polymer and Molecular Electronics (PME) Group, IMEC, Leuven. He is the author or coauthor of about 90 papers in refereed journals. His current research interests are organic and oxide transistors and circuits, as include the characterization, physical modeling, and numerical simulation of electronic devices based on organic and metal-oxide-semiconductors, as well as the design of nonvolatile memory cells in standard CMOS technology. He regularly serves as a reviewer for specialized technical journals.

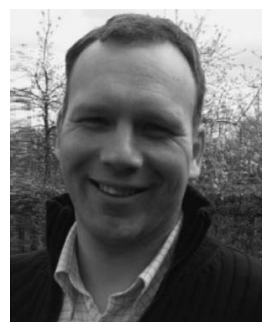
well as organic photovoltaics.

Fabrizio Torricelli was born in Desenzano del Garda, Italy, in 1982. He received the Master's de-
gree (with honors) and the Ph.D. degree in electronics engineering from the University of B
Brescia, Italy, in 2006 and 2010, respectively.

From 2006 to 2010, he was a Consultant with Fellow with the Department of Information Engineering, University of Brescia. Since 2010, he has been with the Eindhoven University of Technology, 


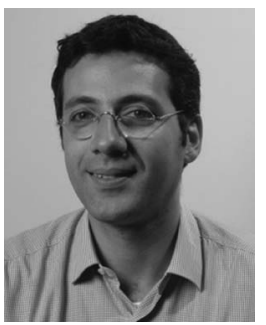

Eugenio Cantatore (M'98) received the Master's and $\mathrm{Ph} . \mathrm{D}$. degrees in electronic engineering from the Politecnico di Bari, Bari, Italy, in 1993 and 1997 respectively.

From 1994 to 1999, he was first a Ph.D. student and then a Fellow at the European Laboratory for Particle Physics (CERN), Geneva, Switzerland. In 1999, he moved to Philips Research, Eindhoven, The Netherlands, as a Senior Scientist. In 2007, he joined the Eindhoven University of Technology, Eindhoven, where he is currently an Associate Professor. He is the author or coauthor more than 80 papers in journals and conference proceedings. He is the holder of 13 patents or patent applications. His research interests include the design and characterization of electronic circuits exploiting emerging technologies such as organic and metal-oxide materials, as well as the design of ultralow-power microsystems for medical applications.

Dr. Cantatore is active in the Technical Program Committees of European Solid-State Device Research Conference, European Solid-State Circuit Research Conference, and International Solid-State Circuit Conference (ISSCC) and is a member of the Executive Committee of ISSCC. He was the recipient of the Beatrice Winner Award for Editorial Excellence from ISSCC in 2006. 